\title{
Optimization Design and Characterization of Helmholtz Coils
}

\author{
Sadiq U. A. ${ }^{1,2,3} \quad$ Oluyombo O. W. ${ }^{1}$ \\ 1.National Space Research \& Development Agency, Abuja, Nigeria \\ 2.Center for Satellite Technology Development, Abuja, Nigeria \\ 3.University of Abuja, Nigeria
}

\begin{abstract}
Earth's magnetic field data from ground-based magnetometer observatories are important for studies related to geomagnetic storm. The absence of earth's magnetic field data observatories results in a complex mysterious phenomena of the geomagnetic storm and remains as unexplained one. Magnetometer is used to monitor and record the earth's magnetic field data at the geomagnetic observatory. It is also used to measure the three components of the field such as the horizontal component $(\mathrm{H})$, the declination component (D), and the downward component $(\mathrm{Z})$. Fluxgate magnetometer is contributing to the ongoing extensive research work dedicated to explanation of some of the complex phenomena related to the geomagnetic storm and solar terrestrial system. In order to examine the magnetic field sensing of a fluxgate sensor, a large area with uniform magnetic field is required. The advantage of having a large area is that it will allow easy access of the sensor during measurement. A laboratory design and characterization of Helmholtz coils is a better choice when Helmholtz coil with larger areas that are available in the market are very expensive. This paper presents the optimization design of Helmholtz coils to create magnetic fields, which could be used to null the earth's magnetic field, calibrate magnetic sensors, and used for other experiments in which a controllable amount of uniform magnetic field is required.
\end{abstract}

DOI: $10.7176 /$ JIEA/9-4-05

Publication date:June $30^{\text {th }} 2019$

\section{I.INTRODUCTION}

Helmholtz coil is a parallel pair of two similar circular coils, spaced one radius apart with coils wound in series such that current passes in same direction in each coil (Abbott, 2015). Helmholtz coils are usually employed to create magnetic fields to null the earth's magnetic field, calibrate magnetic sensors, and used for other experiments in which a controllable amount of uniform magnetic field is required (Abbott, 2015). The basic principle of Helmholtz coils is that the coils produces uniform magnetic field at the center (Abbott, 2015; Daron et al., 2015). This arrangement of coil was invented by German physicist, Hermann von Helmholtz, over a century ago (Bhatt et al, 2010). The intensity of magnetic field is directly proportional to the number of turns and the current through the coils (Abbott, 2015). Starting with the desired dimension based on the radius/coil separation $R$ and magnetic field strength $B$, the required current $I_{C}$ and the number of turn $\mathrm{N}$ can be traded and selected according to the following equation for field strength at the center of the Helmholtz coil (Bhatt et al., 2010):

$$
B=N \cdot \frac{\mu_{0} \cdot I_{C}}{R} \cdot\left(\frac{4}{5}\right)^{3 / 2}
$$

where $R$ is the radius of each turn of a coil, $I_{C}$ is the coil current, and $\mu_{0}$ is the permeability of air. The power supply for driving the coils must also be taken into consideration when calculating the diameter of wire to be used to ensure it can provide the current needed to generate the required magnetic field (Bhatt et al., 2010). The schematic describing the configuration of Helmholtz coils is shown in Figure 1.

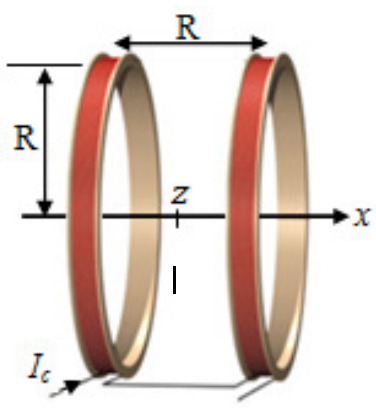

Figure 1: The Schematic Diagram of Helmholtz Coils (Bhatt et al, 2010)

Each of the individual Helmholtz coils produces uniform magnetic field parallel to the axis of the coils (Bhatt et al, 2010), and the two coils form a system such that a controllable amount of uniform magnetic field can be accomplished by controlling the applied current (Abbott, 2015). The uniform field within the coil accounts from the summation of fields parallel to the axis and the difference of vertical component fields (Abbott, 2015). Helmholtz coil design is simple and there are many commercially available designs for all kinds of applications. In order to examine the magnetic field sensing of a fluxgate sensor, a large area with uniform magnetic field is 
required (Butta et al., 2010). The advantage of having a large area is that it will allow easy access of the sensor during measurement (Butta et al., 2010). A laboratory design and characterization of Helmholtz coils is a better choice when Helmholtz coil with larger areas that are available in the market are very expensive.

Hence, there is need for a systematic optimization approach for Helmholtz coils design to find its optimum performance. The combined modified multi-objective FOA and systematic optimization approach is used to improve the Helmholtz coils design in this research by optimizing its magnetic field created by the coils. Such a multi-objective optimization algorithm is powerful in dealing with design problems in electro-magnetics with a large number of design variables and multiple objectives under complex nonlinear constraints.

\section{OPTIMIZATION DESIGN}

The goal of the optimization of Helmholtz coils in this section was to find the optimal number of coils turns and suitable coil geometry of the Helmholtz coils that minimize the driving current coil current and magnetic field of the Helmholtz coils. The algorithm starts by placing the fireflies in random locations. The location of a firefly corresponds to the values of the parameters (number of turns and geometry Helmholtz coils) for the objective function (Coil current and magnetic field) to be solved. Five design variables were identified and are presented in Table 1 . The geometry constraints and operating limits are shown in Table 2.

Table 1: Helmholtz Coils Design Variables and Ranges

\begin{tabular}{llll}
\hline Variables & Range & Unit \\
\hline Coil width turns & $10-50$ & - \\
Coil height turns & $5-50$ & - \\
Wire diameter & $0.135-1.0$ & $\mathrm{~mm}$ \\
Coil radius & $0.1-0.25$ & $\mathrm{~m}$ \\
Coil current & $10-250$ & $\mathrm{~mA}$ \\
\hline
\end{tabular}

Table 2: Helmholtz Coils Design Constraints

\begin{tabular}{l|l|l}
\hline Constraints & Range & Unit \\
\hline Total winding turns & $\leq 2500$ & - \\
Magnetic field & $\leq 500$ & $\mu \mathrm{T}$ \\
\hline
\end{tabular}

The design variables were written as:

$$
X=\left[x_{1}, x_{2}, \ldots x_{5}\right]=\left[\begin{array}{c}
\text { Coil width turns } \\
\text { Coil height turns } \\
\text { Wire diameter } \\
\text { Coil radius } \\
\text { Coil current }
\end{array}\right]^{T}
$$

The geometric constraints and operating limits were represented as:

$$
g(x) \leq 0 \Leftrightarrow\left\{\begin{array}{l}
\text { Total winding turns } \\
\text { Total magnetic field }
\end{array}\right\}
$$

The optimization design problem of the Helmholtz coils was formulated by defining the magnetic field inside the Helmholtz coils as the objective function:

$$
\text { Minimize } B(z)=\frac{\mu_{0} I_{c} \cdot R^{2}}{2} \cdot\left(\begin{array}{c}
\left(\left(z+\frac{R}{2}\right)^{2}+R^{2}\right)^{-\frac{3}{2}} \\
+\left(\left(z-\frac{R}{2}\right)^{2}+R^{2}\right)^{-3 / 2}
\end{array}\right)
$$

The multiple objectives in this research using FOA were implemented based on the following steps:

1. Initialize the number of fireflies, $n$, biggest attraction $\beta_{0}$, absorption coefficient of light intensity $\gamma$, step size factor $\alpha$, and maximum number of iterations or generations $t_{\max }$.

2. Initialize the positions of fireflies randomly, namely initializing design variables of the FMS parameters (magnetic core, pick-up coil, and detection circuit), the values of objective function (noise level) of fireflies are set as their maximum brightness of fluorescence $I_{0}$.

3. Calculate relative brightness and attractiveness of fireflies, which belong to the population. The direction of movement depends on the relative brightness of fireflies. Here (Yang, 2013):

$$
\begin{aligned}
& I=I_{0} \times e^{-\gamma r i j} \\
& \beta=\beta_{0} \times e^{-\gamma r i j}
\end{aligned}
$$

where $I_{0}$ is the maximum fluorescence brightness of the firefly, namely the fluorescence brightness itself $(r=0)$, which depends on the value of the objective function. $\beta_{0}$ is the maximum attractiveness, namely the attractiveness of the light source $(r=0), \gamma$ is the absorption coefficient of the light intensity. The fluorescence will gradually weaken according to the increasing distance and the absorption of media. The 
absorption coefficient of light intensity is set to reflect this feature. $r_{i j}$ is the spatial distance between firefly $i$ and $j$.

4. Update the spatial positions of fireflies. Random perturbations are injected to the firefly with the best position. The updated equation is:

$$
x_{i}=x_{i}+\beta \times\left(x_{j}-x_{i}\right)+\alpha \times(\text { rand }-0.5)
$$

where $x_{i}, x_{j}$ represent the spatial positions of firefly $i$ and $j$, respectively. $\alpha$ is the step size factor. rand is random factor distributed uniformly in $[0,1]$.

5. Recalculate the brightness of fireflies according to the updated positions.

6. Return to Step 3 until the search precision is met or the maximum number of generations is achieved.

\section{III.METHODOLOGY}

The FOA was written in Matlab environment and the results were obtained by running the developed program on a $1.50 \mathrm{GHz}$ Intel ${ }^{\circledR}$ core $^{\mathrm{TM}}$ Duo CPU Windows 7 Ultimate 32-bit personal computer. In this research, the primary objective of the Helmholtz coils designed was to provide uniform magnetic field in the test area. The characterization was carried out using a developed Fluxgate Magnetometer Sensor (FMS) with coil driven by DC source. A low frequency $(50 \mathrm{~Hz})$ Helmholtz coil driver was used to generate the required magnetic field. Since the magnetic field density is proportional to electrical current, to generate high magnetic field, high current is needed. However, at high frequency the Helmholtz coils impedance is also high, which will require high voltage to drive the desired current. For a given driver voltage amplitude, the coil current is inversely proportional to the coil impedance. Therefore the two opposing factors that affect the magnetic field are current and frequency.

Each of the Helmholtz coils was wound with 22 turns coil width and 25 turns coil height as obtained from modified FOA design results. Each coil had a diameter of $12.88 \mathrm{~cm}$ with 550 turns each of copper wire. The copper wire with $0.449 \mathrm{~mm}$ diameter was wound on each coil bobbin. When winding the coils, care was taken to ensure that the windings were in order by exerting pressure on the wire as the winding proceeded, but at the same time ensure that the pressure exerted was not too large as the wire might break, which might be risk after winding 500 turns.

A major problem that was encountered after winding the coil was that the coil forms (bobbin around which the coil was wound) were deformed due to the pressure produced by the tight windings. The form had its original width at the bottom, but as more and more layers of windings were added, the width of the form was expanding. Since the windings were almost finished, there was nothing to do about it. However, for future windings of coils of this kind, the lessons learned from this research was to ensure that the coil forms are made strong enough to resist the pressure of all the windings. Figure 2 shows the final Helmholtz coil structure with test bed at the center.

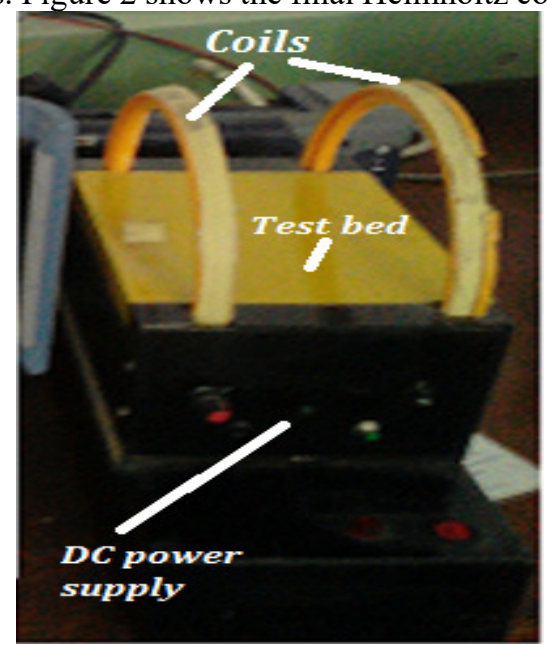

Figure 2: Complete Helmholtz Coils with Test Bed.

After having finished the fabrication of the Helmholtz coils, there was need to characterize the coils to ascertain that the coils work the way the modified FOA predicted. Therefore, the magnetic fields inside the Helmholtz coils were measured as a function of distance $\mathrm{x}$ along the axis of symmetry of the Helmholtz coils (see Figure 1). Furthermore, the magnetic field across the midway point between coils was measured as a function of the current through the coils. The objectives of these measurements were to study and verify sensitivity of the magnetic field of a pair of Helmholtz coils, and also to learn about the rationale for using Helmholtz coils.

In order to measure the magnetic field as a function of distance $\mathrm{x}$ along the axis of symmetry of the Helmholtz coils and when the current through the coils was varied, magnetic field strength meter (Tenmars, model TM-191 portable) was used to measure the DC magnetic field in the coils. Figure 3 shows the block diagram of the apparatus and setup for Helmholtz coils magnetic fields measurements. The actual implementation of the setup is shown in 
Figure 4 .

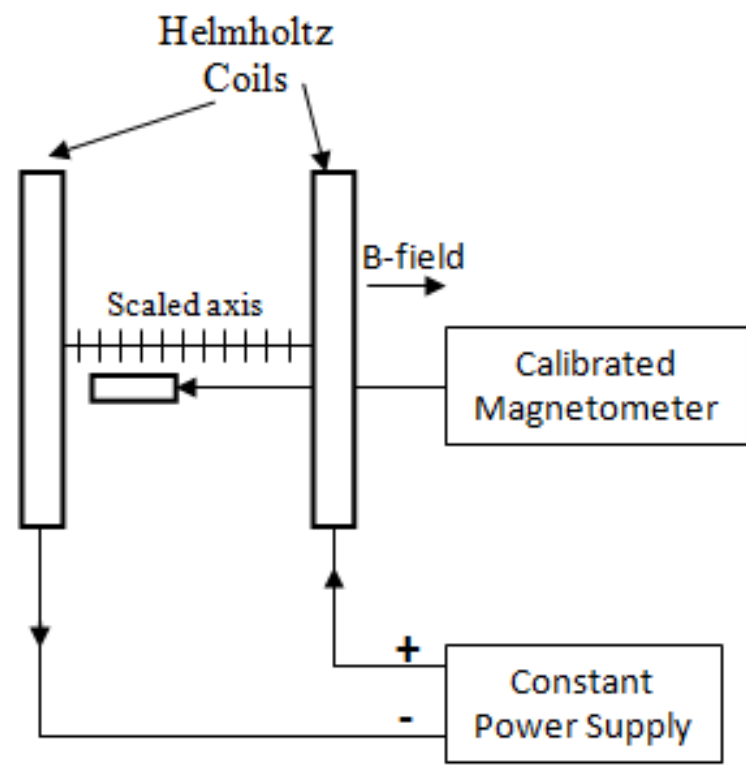

Figure 3: Block Diagram of Experimental Setup for Characterizing the Helmholtz Coils.

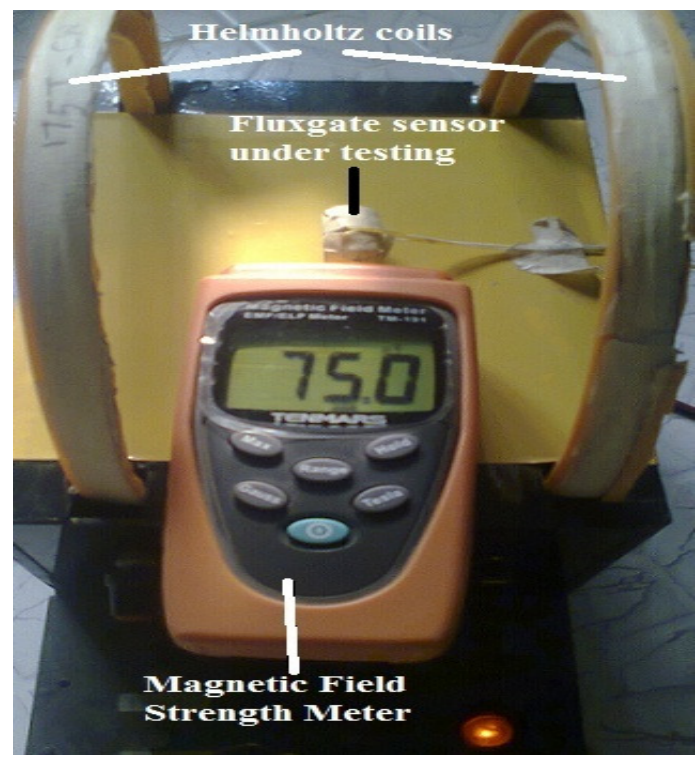

Figure 4: Helmholtz Coils Characterization used as a Test Bed for testing FMS.

As shown in Figure 3, during the measurements, a constant current of $100 \mathrm{~mA}$ (obtained from optimization results) was passed through the coils and read the value of the magnetic field (Figure 4) on a calibrated magnetic field meter (Tenmars, model TM-191 portable) for each $3 \mathrm{~mm}$. The flux density was measured along the horizontal axis (see Figure 1) of the coils. The measured magnetic field was in the range of $180 \mu \mathrm{T}$ with $8 \mu \mathrm{T}$ resolution.

\section{IV.RESULTS AND DISCUSSION}

Table 3 shows the results for the Helmholtz coils design variables. In the Helmholtz coils, the wire size was decreasing and the coil radius was also decreasing in an effort to optimize the magnetic field of Helmholtz coils. Consequently, there was not a significant increase in the dimensions and geometry of the coils between the runs. Table 3: FOA Results for the Helmholtz Coils Simulations.

\begin{tabular}{l|c|c|c|c|c|c}
\hline & \multicolumn{5}{|c|}{ Input variables } & $\begin{array}{l}\text { Output } \\
\text { variable }\end{array}$ \\
\hline $\begin{array}{l}\text { Helmholtz } \\
\text { Coils }\end{array}$ & $\begin{array}{c}\text { Coil width } \\
\text { turns }\end{array}$ & $\begin{array}{c}\text { Coil height } \\
\text { turns }\end{array}$ & $\begin{array}{c}\text { Wire } \\
\text { diameter } \\
(\mathbf{m m})\end{array}$ & $\begin{array}{c}\text { Coil radius } \\
(\mathbf{m m})\end{array}$ & $\begin{array}{c}\text { Coil current } \\
(\mathbf{m A})\end{array}$ & $\begin{array}{l}\text { Magnetic } \\
\text { field }(\boldsymbol{\mu} \mathbf{T})\end{array}$ \\
\hline FOA Design & 22 & 25 & 0.449 & 12.88 & 100 & 332 \\
\hline
\end{tabular}

Matlab programs were written to obtain the magnetic field. The measured and calculated magnetic fields of 
the Helmholtz coils for $100 \mathrm{~mA}$ of DC current through the coils were presented in Table 4.

Table 4: Measurements and Calculated Magnetic Field of Helmholtz Coils

\begin{tabular}{l|c|c|c|c|c}
\hline $\begin{array}{l}\text { Driving } \\
\text { current } \\
(\mathbf{m A})\end{array}$ & $\begin{array}{c}\text { Calculated } \\
\text { magnetic field }\end{array}$ & $\begin{array}{c}\text { Measured } \\
\text { magnetic field }\end{array}$ & $\begin{array}{l}\text { Driving } \\
\text { current } \\
\text { (mA) }\end{array}$ & $\begin{array}{c}\text { Calculated } \\
\text { magnetic field }\end{array}$ & $\begin{array}{l}\text { Measured } \\
\text { magnetic field }\end{array}$ \\
\hline 2 & 3.62 & 0.42 & 52 & 94.06 & 94.06 \\
4 & 7.24 & 1.24 & 54 & 97.68 & 95.68 \\
6 & 10.85 & 6.05 & 56 & 101.30 & 101.30 \\
8 & 14.47 & 8.14 & 58 & 104.92 & 104.92 \\
10 & 18.09 & 18.09 & 60 & 108.54 & 106.54 \\
12 & 21.71 & 21.71 & 62 & 112.15 & 112.15 \\
14 & 25.32 & 25.32 & 64 & 115.77 & 114.27 \\
16 & 28.94 & 28.94 & 66 & 119.39 & 118.99 \\
18 & 32.56 & 32.56 & 68 & 123.01 & 124.16 \\
20 & 36.18 & 36.18 & 70 & 126.62 & 127.98 \\
22 & 39.80 & 39.80 & 72 & 130.24 & 131.77 \\
24 & 43.41 & 43.39 & 74 & 133.86 & 134.60 \\
26 & 47.03 & 47.20 & 76 & 137.48 & 136.64 \\
28 & 50.65 & 50.22 & 78 & 141.10 & 140.56 \\
30 & 54.27 & 54.97 & 80 & 144.71 & 143.97 \\
32 & 57.89 & 57.88 & 82 & 148.33 & 147.29 \\
34 & 61.50 & 61.56 & 84 & 151.95 & 150.97 \\
36 & 65.12 & 65.72 & 86 & 155.57 & 154.91 \\
38 & 68.74 & 68.57 & 88 & 159.19 & 156.39 \\
40 & 72.36 & 72.66 & 90 & 162.80 & 163.72 \\
42 & 75.97 & 76.71 & 92 & 166.42 & 168.38 \\
44 & 79.59 & 80.91 & 94 & 170.04 & 170.34 \\
48 & 83.21 & 84.21 & 96 & 173.66 & 174.55 \\
50 & 86.83 & 87.03 & 98 & 180.89 & 176.23 \\
\end{tabular}

Magnetic field plot arising from the contributions of the designed coils is shown in Figure 5.

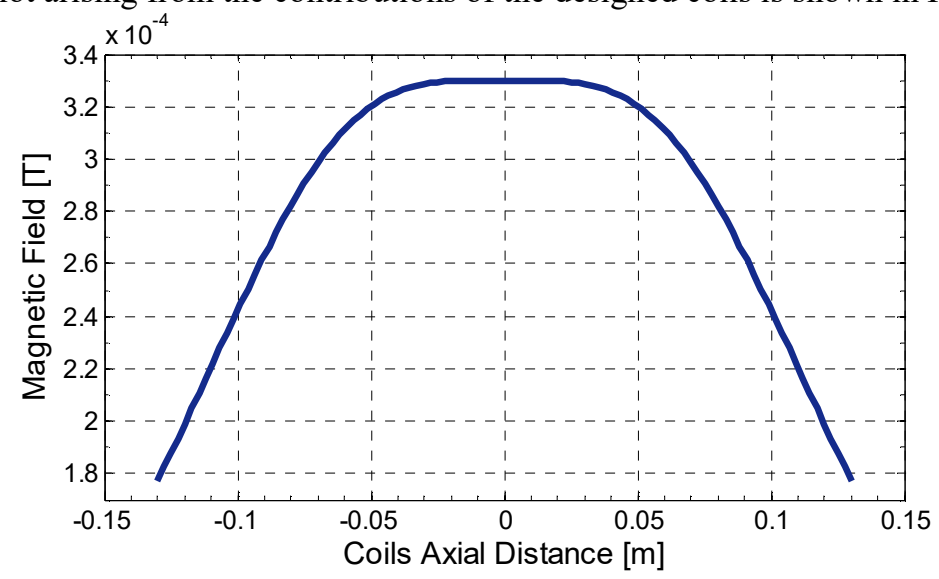

Figure 5: Axial Magnetic Field against Distance for Helmholtz Coils FOA Design.

The primary difference between the Helmholtz coils and FMS is that the Helmholtz coils uses a pair of similar coils to generate uniform magnetic field while the FMS is used to detect the magnetic field created by the Helmholtz coils. It can be concluded that the magnetic field is very homogeneous as long as the shape of the coils is approximately a square. Therefore, any deviation from the optimum coil configuration would cause the graph to be in different form. The values of the measured magnetic field plotted as a function of axial distance is shown in Figure 6. 


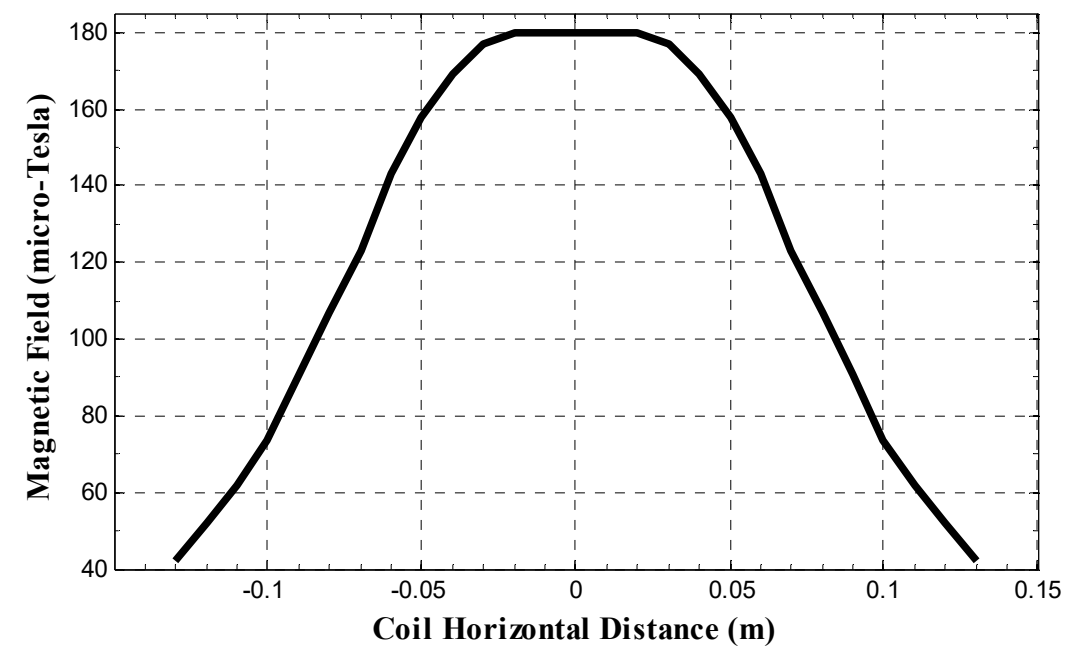

Figure 6: Measured Magnetic Field of Helmholtz Coils.

As seen in Figure 6, the measured magnetic flux density was far from the simulated value because of the limited range of the calibrated magnetometer employed $(0 \mu \mathrm{T}$ to $200 \mu \mathrm{T})$. The measured flux density along the axis of the coil was symmetric about the center. This showed that the coils were aligned perfectly and the magnetic flux density from the individual coils was symmetric.

To investigate the dependence of magnetic field at the center point (where the magnetic field was peak) of Helmholtz coils on the applied driving current, a calibrated magnetometer was placed at the centre point (where the magnetic field was peak) of the coils. A measurement of the magnetic field was made for each $2 \mathrm{~mA}$ in the range of $100 \mathrm{~mA}$. The measurements and calculated values were plotted as shown in Figure 7.

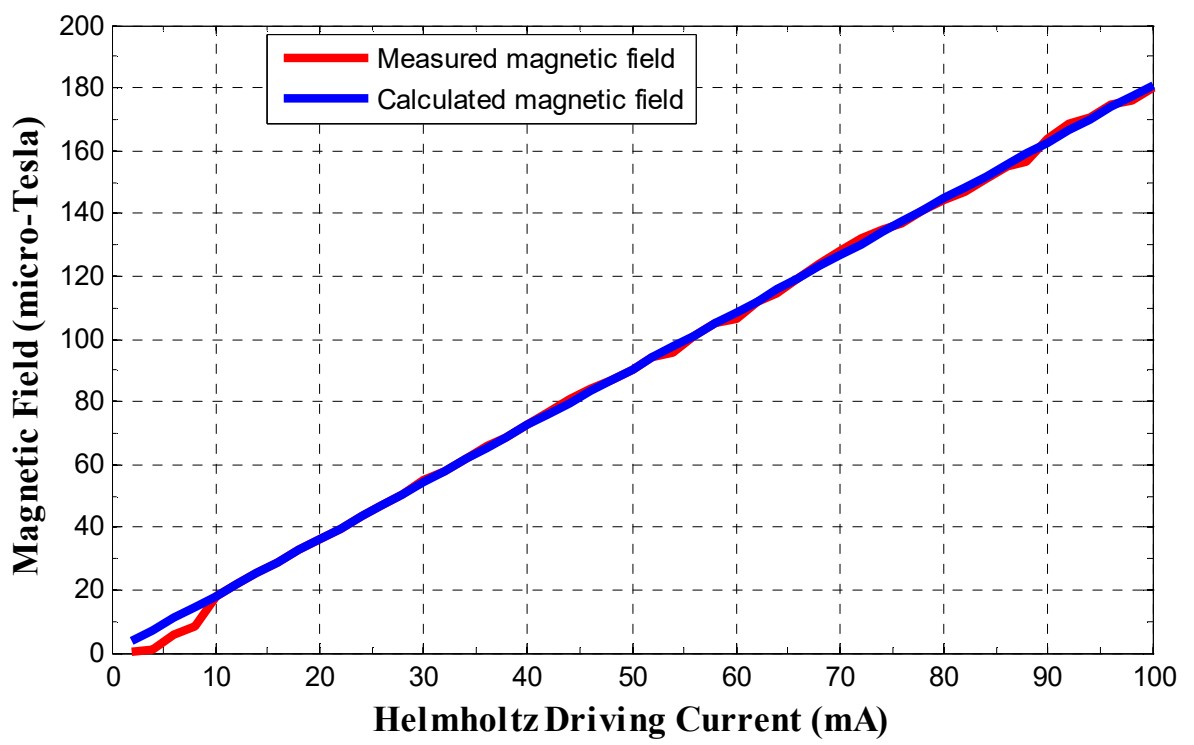

Figure 7: The Magnetic Field in the Center Point of Coils as a Function of Current.

The figure showed that the measurements agreed with the calculations. However, it was observed that the magnetic field measurements were not consistent at the current below $10 \mathrm{~mA}$ which resulted in the magnetic field not also consistent below $10 \mu \mathrm{T}$. Generally, the inconsistencies in the measurements could be due to the interference of the earth's magnetic field or the presence of other magnetic field sources such as phones, laptops, buckle from a belt, and other. The presence of magnetic interference could cause increase or decrease of the magnetic field of a sensor and even the Helmholtz coils. Decrease in the magnetic field signifies interference from negative magnetic field while increase in magnetic field signifies positive magnetic interference. Also, the calibrated magnetometer used had a measurement accuracy of $\pm 3 \%$ and it was as well not sensitive to very low magnetic field. In addition, it was suspected that another reason for the drift of the measurements in comparison to the calculated values was the heat, which was produced in the Helmholtz coils material when current was run through the coils. 


\section{CONCLUSION}

The primary difference between the Helmholtz coils and FMS is that the Helmholtz coils uses a pair of similar coils to generate uniform magnetic field while the FMS is used to detect the magnetic field created by the Helmholtz coils. With the coil parameters obtained from modified FOA designs of the Helmholtz coils, it can be concluded that the magnetic field is very homogeneous as long as the shape of the coils is approximately a square. Therefore, any deviation from the optimum coil configuration would cause the graph to be in different form.

The measured magnetic fields of the Helmholtz coils for $100 \mathrm{~mA}$ of DC current through the coils were presented. The measured magnetic field was in the range of $180 \mu \mathrm{T}$ with $8 \mu \mathrm{T}$ resolution.

\section{REFERENCES}

Abbott, J. J. (2015). Parametric design of tri-axial nested Helmholtz coils. Review of Scientific Instruments, 86/110 .

Bhatt, V.; Rautela, R.S.; Sharma1, P.; Tiwari, D.C. and Khushu, S. (2010). Design and development of Helmholtz coil for hyperpolarized MRI. Proceedings of the COMSOL Conference, 1-6.

Butta, M.; Ripka, P.; Infante, G.; Badini-Confalonieri G.A., and Vázquez, M. (2010). Magnetic microwires with field induced helical anisotropy for coil-less fluxgate. IEEE Transactions on Magnetics, 46(7)/2562-2565.

Daron, B.B.; Jonathan, E. and Madsen, M.J. (2015). Magnetic field mapping. Wabash Journal of Physics (WJP), $4(3) / 1-13$. 